\title{
Linking Population Dynamics to Microbial Kinetics for Hybrid Modeling of Engineered Bioprocesses
}

Zhang Cheng ${ }^{\text {a }}$, Shiyun Yao ${ }^{\text {a, }}$ Heyang Yuan ${ }^{\mathrm{a}, *}$.

${ }^{a}$ Department of Civil \& Environmental Engineering, Temple University, 1947 N. 12th Street, Philadelphia, PA 19122, USA

Type of contribution: Research Article

\section{* Corresponding author}

heyang.yuan@temple.edu; 


\section{Abstract}

2 Mechanistic and data-driven models have been developed to provide predictive insights into the

3 design and optimization of engineered bioprocesses. These two modeling strategies can be

4 combined to form hybrid models to address the issues of parameter identifiability and prediction

5 interpretability. Herein, we developed a novel and robust hybrid modeling strategy by

6 incorporating microbial population dynamics into model construction. The hybrid model was

7 constructed using bioelectrochemical systems (BES) as a platform system. We collected 77

8 samples from 13 publications, in which the BES were operated under diverse conditions, and

9 performed holistic processing of the 16S rRNA amplicon sequencing data. Community analysis

10 revealed core populations composed of putative electroactive taxa Geobacter, Desulfovibrio,

11 Pseudomonas, and Acinetobacter. Primary Bayesian networks were trained with the core

12 populations and environmental parameters, and directed Bayesian networks were trained by

13 defining the operating parameters to improve the prediction interpretability. Both networks were

14 validated with Bray-Curtis similarly, relative root-mean-square error (RMSE), and a null model.

15 The hybrid model was developed by first building a three-population mechanistic component and

16 subsequently feeding the estimated microbial kinetic parameters into network training. The hybrid

17 model generated a simulated community that shared a Bray-Curtis similarity of $72 \%$ with the

18 actual microbial community and an average relative RMSE of $7 \%$ for individual taxa. When

19 examined with additional samples that were not included in network training, the hybrid model

20 achieved accurate prediction of current production with a relative error-based RMSE of 0.8 and

21 outperformed the data-driven models. The genomics-enabled hybrid modeling strategy represents

22 a significant step toward robust simulation of a variety of engineered bioprocesses. 
bioRxiv preprint doi: https://doi.org/10.1101/2021.04.15.440059; this version posted April 15, 2021. The copyright holder for this preprint (which was not certified by peer review) is the author/funder. All rights reserved. No reuse allowed without permission.

24 Keywords: Engineered bioprocess, Microbial population dynmics; Microbial kinetics; Machine

25 learning, Bayesian network; Hybrid modeling. 


\section{Introduction}

27 Engineered bioprocesses are widely applied to treat waste streams and recover valuable resources

28 (Rittmann and McCarty 2012). To facilitate the design and optimization of full-scale bioprocesses,

29 a number of mechanistic and data-driven models have been developed over the past 60 years

30 (Batstone et al. 2002, Bhat and McAvoy 1990, Henze et al. 2000).

32 Mechanistic models can provide predictive insights into the fundamental processes in biological

33 systems (Jeppsson 1996). The model structure has been improved with a greater understanding of

34 the microbiomes in biological systems (Donoso-Bravo et al. 2011, Henze et al. 2000, Ng and Kim

35 2007). For example, the Anaerobic Digestion Model No.1 composed of 19 bioconversion steps

36 and 100 parameters is by far the most comprehensive mechanistic model formulated for engineered

37 bioprocesses and can be readily modified to simulate specific applications (Liu et al. 2017,

38 Rodríguez et al. 2008, Zhao et al. 2019). An inherent problem of this modeling strategy is that the

39 model structure and parameters are largely unidentifiable (Donoso-Bravo et al. 2011, Jeppsson

40 1996). This stems from the simulation of microbial kinetics, in which the functional populations

41 cannot be fully recapitulated by the Monod expressions, and the associated kinetic parameters

42 cannot be directly measured (Donoso-Bravo et al. 2011, Ng and Kim 2007). Although kinetic

43 parameters can be derived from biochemical measurements, they show considerable variations

44 under different operating conditions (Bernard et al. 2006, $\mathrm{Ng}$ and Kim 2007). As a result, a

45 mechanistic model developed for a specific bioprocess needs constant parameter calibration to

46 cope with operational perturbations but still falls short when applied to other biological systems. 
Data-driven models are not limited by identifiability issues and can yield more accurate predictions than mechanistic models do when a sufficiently large data pool is provided (Walpole et al. 2017). Artificial neural networks can be constructed with appropriate input variables and network architecture to predict the effluent quality (Mendes et al. 2015, Moral et al. 2008). Recent studies

52 have demonstrated the applicability of several machine learning algorithms, including support

53 vector machine, random forest, extreme gradient boosting, and k-nearest neighbors, to full-scale

54 anaerobic digesters (De Clercq et al. 2019, Wang et al. 2020b). Despite the outstanding learning

55 performance, most of the data-driven models are black boxes that are unable to generate

56 interpretable predictions (Rudin 2019). This is particularly problematic for complex biological

57 systems whose performance is largely determined by the microbial population and activity, and

58 thus no mechanistic insights can be obtained from the simulation. Training data-driven models

59 with microbial population dynamics presents a promising solution to tackle this issue. Previous

60 studies have incorporated genomic data into machine learning models (neural networks and

61 Bayesian networks) to reconstruct microbial communities in natural ecosystems (Kuang et al. 2016,

62 Larsen et al. 2012). Using similar strategies, an array of data-driven models was constructed to

63 simulate the performance and stability of engineered bioprocesses (Lesnik et al. 2020, Lesnik and

64 Liu 2017, Yuan et al. 2017).

Hybrid models can potentially address the limitations of those two modeling strategies (Cote et al.

67 1995, Karama et al. 2001, Zhao et al. 1997). A common approach is to couple the mechanistic and

68 statistical components in series. The error signals obtained from the mechanistic component are

69 converted into those for the network component, which are subsequently used to update the

70 network weights through back-propagation (Lee et al. 2002). Through such integration, hybrid 
71 models yield robust and semi-interpretable predictions for non-linear behaviors such as microbial

72 kinetics (Zendehboudi et al. 2018). By far, all hybrid models are built with physical and

73 biochemical parameters and thus unable to reveal the connections between microbial population

74 and microbial kinetics. We propose to link them and improve the prediction robustness by

75 incorporating genomic data into model construction.

77 The objectives of this study are to 1) comprehensively characterize the microbiome in a specific

78 type of bioprocess and 2) formulate a novel hybrid model based on the population dynamics and

79 microbial kinetics. To this end, we used bioelectrochemical systems (BES) as a platform system

80 for model construction. As emerging biotechnology that simultaneously achieves

81 water/wastewater treatment and energy/resource recovery (Logan et al. 2006, Wang and Ren 2013),

82 BES are ideal for this task because they respond quickly to environmental perturbations (Yuan et

83 al. 2016, Yuan et al. 2015), with current production acting as a sensitive indicator of the microbial

84 population and functional dynamics. The microbial communities in BES are highly enriched with

85 relatively low microbial diversity (Yates et al. 2012, Zhu et al. 2014), and hence represent a

86 desirable level of complexity: diverse enough to be relevant to the microbiomes in other

87 bioprocesses yet simple enough to be in silico reconstructed. To improve the compatibility of our

88 models, we performed an extensive literature review and collected the 16S rRNA gene amplicon

89 sequencing data from 77 samples in 13 publications, in which the BES were operated under a wide

90 range of conditions. Core populations were selected at different taxonomic levels and used to train

91 Bayesian networks, a machine learning model capable of characterizing the causal relationships

92 among variables (Uusitalo 2007). Meanwhile, microbial kinetics were calculated using a three-

93 population mechanistic component and fed into the training process to improve the prediction. 
94 This hybrid modeling strategy is expected to take advantage of the rapid growth of the genomic

95 database (Kahn 2011), circumvent the time-consuming calibration of the kinetic parameters, and

96 be broadly applicable to a variety of engineered bioprocesses.

97

\section{Materials and Methods}

$99 \quad 2.1$ Data collection and sequence processing

100 A comprehensive literature review was carried out, and 13 publications containing 77 samples

101 were selected for downstream community analysis and model construction. The detailed

102 information about the selected publications is listed in the Supporting Information (SI) Table S1.

103 The selection criteria are: 1) 16s rRNA gene amplicon sequences were properly deposited in the

104 National Center for Biotechnology Information (NCBI) or DNA Data Bank of Japan (DDBJ)

105 databases for holistic sequence processing; 2) the results reported eight key parameters closely

106 related to BES performance, including substrate composition and concentration, coulombic

107 efficiency (CE), pH, current, anode area, external resistance, hydraulic retention time, and

108 temperature; 3 ) the results included the variation of chemical oxygen demand (COD) over time or

109 currents/voltage that can be used to calculate the time series of COD. The selected studies show a

110 variety of reactor configurations, operation modes, substrates, and operating conditions (SI Table

$111 \mathrm{~S} 1$ ), which is expected to enhance the compatibility of the predictive models developed in the

112 present study.

113

114 The sequence data from the selected publications contained both pair-end or single-end reads and

115 were converted into a uniform format before further processing. Briefly, pair-end reads were

116 merged using Vsearch (Rognes et al. 2016), and the chimeric and low-quality sequences were 
117 eliminated using the QIIME2 plug-in DADA2 (Callahan et al. 2016). The Greengenes database

118 (gg_13_8 updated February 2011) was used to conduct sequence alignment and train the taxonomy

119 classifier of the denoised sequences (DeSantis et al. 2006). In addition, due to the various primer-

120 targeted regions (V1-V9) used to amplify 16s rRNA gene in those studies, the primer pair 8F/907R

121 was set as the forward and reverse primers for the classifier to encompass all of the sequences from

122 the samples.

\section{$124 \quad 2.2$ Community analysis}

125 Alpha (Shannon and Simpson indices) diversity analysis, principal coordinate analysis (PCoA), 126 and redundancy analysis (RDA) were performed using R to unravel intra-sample diversity and

127 inter-sample distance. Core populations were selected at the genus, order, and phylum levels with

128 the following criteria (Yuan et al. 2019): 1) at least one occurrence in the 13 studies with the 129 relative abundance $\geq 0.05 \%$ and 2 ) average relative abundance $\geq 2 \%$ across all 77 samples. The 130 criteria allow us to retain the major functional populations in the microbial community and 131 compress the genomic data for downstream model construction. For this reason, core population 132 was not selected at the operational taxonomic unit (OTU) level as the $100 \%$-similarity clustering 133 strategy generated over 9,000 OTUs, and the majority of the samples have little overlap on the 134 community composition. To build a phylogenetic tree for the core genera, the sequence of the most 135 abundant OTU within a core genus was selected as a representative. The phylogenetic tree was 136 built using ARB (Ludwig et al. 2004), and the Silva database (LTPs132_SSU.arb for 16s rDNA 137 updated June 2018) was used for sequence alignment (Quast et al. 2013).

\subsection{Bayesian network analysis}


140 To prepare for network construction, the abundance of the core taxa and the values of the

141 environmental parameters were scaled to $0-1$ (Bishop 2013):

$142 \quad w_{n, i, j}=\frac{w_{i, j}-w_{j, \min }}{w_{j, \max }-w_{j, \min }}($ Eq. 1$)$

143 where $w_{i, j}$ is the relative abundance of population $j$ in sample $i, w_{j, \max }$ is the maximum relative

144 abundance of population $j, w_{j, \min }$ is the minimum relative abundance of population $j$, and $w_{n, i, j}$ is

145 the normalized abundance of population $j$ in sample $i$. Structure learning and parameter learning 146 were performed using the hill-climbing algorithm and maximum likelihood estimation, 147 respectively (Scutari 2010).

149 Primary networks (SI Figures S4A, S5A, and S6A) were trained without considering the $a$ priori

150 knowledge about the operating conditions, and the node directions were solely inferred by the 151 network algorithm. Directed networks (SI Figures S4B, S5B, and S6B) were trained by defining 152 temperature, anode electrode area, external resistance, and hydraulic retention time as the parent 153 nodes. This was because those parameters remained unchanged throughout the operation and thus 154 were not affected by other parameters. A blacklist function was applied to define the unidirectional 155 relationships between those operating parameters and other variables. The same training 156 procedures were conducted at the genus (38 core taxa), order (32 core taxa), and phylum (13 core 157 taxa) levels. Considering the sample quantity and computational cost, a leave-one-out cross158 validation strategy was selected for three validation methods (Bro et al. 2008): Bray-Curtis 159 similarity between the predicted and observed microbial community, relative root-mean-square 160 error (RMSE, Eq. 2), and null model analysis.

relative $R M S E=\frac{\sqrt{\frac{\sum_{1}^{n}\left(\hat{y}_{i}-y_{i}\right)^{2}}{n}}}{y_{\max }}(\mathrm{Eq} .2)$ 
162 where $\hat{y}_{i}$ is the predicted value; $y_{i}$ is the observed value; $y_{\max }$ is the maximum observed value;

163 and $n$ is the number of samples. Null models were constructed by setting the abundance of all core

164 taxa to the average abundance across all samples (Gotelli 2002 ).

165

\subsection{Hybrid model construction}

167 A hybrid model was constructed at the genus level to bridge population dynamics and microbial

168 kinetics following the procedures shown in SI Figure S1. Briefly, maximum substrate utilization

169 rates, maximum growth rates, and mediator yield were calculated using the mechanistic

170 component described below and included as the nodes for network training. Although these kinetic

171 parameters are unmeasurable, the normalization step (Eq. 1) converts exact values into a general

172 tendency of microbial activity that can be statistically connected to the actual relative abundance

173 of the core population (Weissman et al. 2021), and thus the estimated kinetic parameters do not

174 need to be validated.

175

176 The mechanistic component assumes two-step degradation (SI Figure S2) of the substrates by three

177 populations (Pinto et al. 2011). At the first stage, complex organic matters such as polysaccharides,

178 lipids, and proteins are decomposed by primary degraders to low-molecular intermediate products

179 including acetic acid, propionic acid, ethanol, etc. At the second stage, electroactive and non-

180 electroactive microbes convert the intermediates into electrical energy and methane, respectively.

181 Take electroactive microbes as an example, the mass balance for the growth and activity are

182 described using Eq. 3 and Eq. 4, respective:

$183 \frac{d X_{e e}}{d t}=\mu_{e e} \frac{S_{1}}{K_{e e}+S_{1}} \frac{M_{o x}}{K_{M}+M_{o x}} X_{e e}-k_{d e e} X_{e e}-D \frac{1+\tanh \left(f_{e e}\left(X_{e e}+X_{n e}-X_{e e, \max }\right)\right)}{2} X_{e e}($ Eq. 3) 
$184 \frac{d M_{o x}}{d t}=-Y_{M} \cdot k_{e e} \frac{S_{1}}{K_{e e}+S_{1}} \frac{M_{o x}}{K_{M}+M_{o x}}+\frac{\gamma \cdot I}{V_{a} \cdot F \cdot X_{e e} n_{e}}$ (Eq. 4)

185 where $X_{e e}$ and $M_{o x}$ are the concentrations of electroactive microbes and mediator, respectively;

$186 K_{e e}$ and $K_{M}$ are the substrate half-saturation constant and mediator half-saturation constant,

187 respectively; $\mu_{e e}$ and $k_{d, e e}$ are the growth and decay rates, $X_{e e, \max }$ is the maximum capacity of

188 electroactive microbes in the anode; $Y_{M}$ is the mediator yield; $\gamma$ is the molecular mass of mediator;

$189 I$ is the current production; $V_{a}$ is the anode volume; $F$ is the Faraday constant $(\mathrm{A} / \mathrm{d} \cdot \mathrm{mol})$; and $n_{e}$ is

190 the number of electrons transfer. The mass balance was modified based on the multiplicative

191 Monod expressions from previous studies (Ping et al. 2014, Pinto et al. 2010). The detailed

192 formulation of the mechanistic component and the parameters can be found in the SI Method and

193 Table S2. Because the selected publications provided multiple types of data and operated the

194 reactors under distinct conditions, the mechanistic component was slightly modified for individual

195 reactors (SI Table S3), and Literature \#27 (S27) was not considered for mechanistic modeling

196 because a time series of COD was not available.

198 The maximum substrate utilization rate and maximum growth rate are considered to be the most

199 critical values for simulation of engineered bioprocesses (Rittmann and McCarty 2012), and

200 mediator yield is a unique parameter for BES. Those kinetic parameters were estimated with

201 specific limits according to previous studies while others parameters were retrieved from literature

202 (Kato Marcus et al. 2007, Wilson and Kim 2016). To estimate the parameters, the total substrate

203 concentration over time and the initial values of the kinetic parameters were collected, calculated,

204 or estimated from the selected papers. Because biomass and mediator concentrations were not

205 measurable and unavailable in the literature, some assumptions are made: 1) primary degraders

206 have an initial concentration of $100 \mathrm{mg} / \mathrm{L}$ in all reactors, 2) electroactive and non-electroactive 
207 microbe grow evenly on the anode surface at an average thickness of $60 \mu \mathrm{m}$ (Lee et al. 2009,

208 Torres et al. 2008), 3) the maximum biofilm capacity in BES was assumed to be $600 \mathrm{mg} / \mathrm{L}$ (Ping

209 et al. 2014), and 4) the electroactive microbe fraction is proportional to the CE. For microbial

210 electrolysis cells whose CE was higher than $100 \%$ because of the applied voltage, the

211 concentration of electroactive microbes was assumed to be $500 \mathrm{mg} / \mathrm{L}$. Because the dimension of

212 the anode electrode was not directly provided in some of the studies, the area was estimated based

213 on the specific area and size of the electrode (Logan et al. 2007).

\section{$215 \quad 2.5$ Model prediction}

216 Six additional publications were collected to further demonstrate the robustness of the hybrid 217 model (SI Table S7). In the first step, the operating parameters (i.e., temperature, anode electrode 218 area, external resistance, and hydraulic retention time) and $\mathrm{pH}$ from those studies were input into 219 the Bayesian networks to predict current and CE directly. In the second step, the kinetic parameters 220 obtained from the Bayesian network were input into the mechanistic component to calculate the 221 steady-state COD, which was then converted into current production based on the expression of 222 CE (Logan et al. 2006). The predicted and observed values were compared using an RMSE based 223 on relative errors (Walpole et al. 2017):

224 relative error based $R M S E=\sqrt{\frac{\sum_{1}^{n}\left(\frac{\hat{y}_{i}-y_{i}}{y_{i}}\right)^{2}}{n}}$ (Eq. 5)

\section{3.1 BES operated under a variety of conditions}


228 The 13 selected studies contained 39 samples from microbial fuel cells, 31 samples from microbial

229 electrolysis cells with external voltage input, and 7 samples from microbial desalination cells with

230 saline environments (SI Table S1). In addition to different reactor configurations, the bioreactors

231 were fed with a variety of substrates whose main organic matter could be categorized as non-

232 fermentable (acetate and methanol), fermentable (glucose, ethanol, and propyl alcohol), or

233 complex (brewery wastewater, food waste, and pig slurry). The selected studies also presented

234 multiple operation modes including batch, continuous, and continuous with pulse substrate loading.

235 In terms of the performance, the 77 samples showed significant difference (SI Table S4) in CE

236 which ranged from $0.02 \%$ (due to high external resistance, e.g., S22) to $140 \%$ (due to applied

237 voltage, e.g., S37). Overall, the selected samples have included the conditions commonly found in

238 BES studies and are expected to yield representative core populations and models.

\section{$240 \quad 3.2$ Core population in BES}

241 The 2.6 million sequence reads from the 77 samples resulted in approximately 9600 OTUs, based

242 on which the alpha diversity was analyzed. Although the diversity indices (Shannon, Simpson, and

243 Chao1, SI Table S5) did not follow a normal distribution as revealed by the Shapiro-Wilk analysis

244 results $(\mathrm{p}<0.05)$, it could well reflect the variation in operating conditions. For example, the

245 Shannon and Simpson indices for the samples fed with complex substrates (i.e., S15, S17, S20,

246 S27, and S42) were 4.25 and 0.96 , respectively, significantly higher than the 2.24 and 0.73 of the

247 samples fed with non-fermentable substrates. Similar results were reported in previous studies

248 (Wang et al. 2020a), and a highly diverse microbial community was expected to enhance system

249 stability (Girvan et al. 2005). In addition, the majority of the samples fed with non-fermentable

250 and fermentable substrates showed a Chao1 index of approximately 100, indicating that those 
251 BESs were sufficiently sampled, and the key microbes could be captured when selecting the core

252 population. This is confirmed by the rarefaction curves presented in some of the selected

253 publications.

255 PCoA based on Bray-Curtis distance showed a critical role of substrate composition in microbial

256 community assembly (Figure 1). Specifically, samples cultivated with starch- and yeast extraction-

257 based synthetic wastewater (S20 and S22) were found in the top right corner of the PCoA graph,

258 while those with complex food waste, brewery wastewater, and pig slurry (S15, S17, S42, and S51)

259 were clustered in the center. S48 used ethanol as the sole carbon source and was isolated from

260 other samples. Additionally, the anode area appeared to be an important factor that drove the

261 microbial community assembly in S48 (SI Figure S3), which was amended with granular activated

262 carbon in the anode. Another key deterministic factor of microbial community assembly is the

263 seed source. Unlike other studies, S49 was inoculated with activated sludge and formed a distinct

264 community structure. Similarly, activated sludge was the seed of S35 and together with

265 temperature (SI Figure S3) led to communities significantly different from other studies. In

266 summary, varied substrate composition, reactor configuration, and operation mode provided a

267 comprehensive pool of microbial communities for model construction.

269 Core populations were selected at different taxonomic levels based on the occurrence (at least one

270 occurrence in the 13 studies with the abundance $\geq 0.05 \%)$ and abundance $(\geq 2 \%$ across all 77

271 samples) (Ling et al. 2016, Saunders et al. 2016). At the genus level, 38 core taxa were identified,

272 accounting for $55 \%$ of the abundance on average. The selection criteria were considered stringent

273 given that the bioreactors were operated under distinct conditions, and the microbial communities 
274 were highly diverse. This was reflected by the loss of several abundant taxa in specific BES. For

275 instance, the core genera made up of less than $20 \%$ of the abundance in some of the samples due

276 to the unique flow pattern (plug-flow, S22), substrate (propyl alcohol, S27), and reactor

277 configuration (applied voltage, S37). Nonetheless, the core population included some well-

278 characterized genera such as Geobacter, Desulfovibrio, Pseudomonas, and Acinetobacter, which

279 were frequently found abundant in BES and potentially involved in current production.

281 The presence of Geobacter often serves as the indicator to explain the BES performance, in 282 particular current production, because a few members of this genus are highly efficient in 283 extracellular electron transfer (EET) (Logan et al. 2019, Lovley et al. 2011). Indeed, Geobacter 284 was identified to be a core taxon (G29, Figure 2) with an average abundance of $15 \%$ across all 285 samples and an individual abundance higher than $2 \%$ in 39 samples. This genus was dominant in 286 S20 (17\%), S35 (71\%), S48 (27\%), and some of the samples in S15 (9\%) and S49 (8\%), which all 287 showed a CE over 15\%. However, Geobacter was not present in other high-CE samples likely 288 because the operating conditions (e.g., high salinity in S12 and S54) did not favor its growth 289 (Miyahara et al. 2015). Desulfovibrio spp. from the same class of Deltaproteobacteria are common 290 sulfate-reducing bacteria whose EET ability has also been reported (Aulenta et al. 2012, Gacitúa 291 et al. 2014, Yu et al. 2011). This genus (G28, Figure 2) was abundant in 13 samples (>2\%) and 292 dominant in ethanol-fed S48 (27\%). Desulfovibrio is known to oxidize ethanol with sulfate as the 293 electron acceptor. In the absence of sulfate, Desulfovibrio can still grow syntrophically with 294 methanogens and oxidize the ethanol to acetate through interspecies hydrogen transfer (Hensgens 295 et al. 1993, Kremer et al. 1988). 
Pseudomonas, a well-studied genus forming biofilm in many anaerobic environments, is another

298 core taxon that has been reported to carry out EET by using phenazines as an electron shuttle

299 (Rabaey et al. 2004). Pseudomonas (G33, Figure 2) was widely present in S12, S15, S22 S35, S49,

300 and S54. This genus potentially plays a critical role in shaping the microbial community structure

301 as the phenazines actively produced for quorum sensing can be scavenged for electron shuttling

302 by other species such as Acinetobacter. As shown in Figure 2, Acinetobacter (G32) was found in

303 samples where Pseudomonas was abundant and was previously speculated to utilize phenazines

304 as electron shuttles for EET (Liu et al. 2013, Yuan et al. 2017). Because EET via electron shuttles

305 is limited by diffusion and the conductivity of the anolyte (Torres et al. 2010), Pseudomonas and

306 its metabolic partners are less ubiquitous than Geobacter in BES, and Pseudomonas-dominated

307 communities are more likely to be found in specific environments such as microbial desalination

308 cells with a high ion concentration (Yuan et al. 2017).

310 Several genera from the class Bacteroidia were found to be abundant (Figure 2). Previous studies

311 suggested that this group of bacteria could degrade complex organic compounds including proteins,

312 polysaccharides, and pectins (Dongowski et al. 2000, Grenier et al. 1989). For instance,

313 Parabacteroides (G6 \& G11, Figure 2) show an abundance higher than 5\% in most of the samples

314 fed with complex organic. It has also been reported that some members in the class Bacteroidia

315 degrade biomass and can serve as scavengers of dead cells (Madigan 2014, Reichenbach 1992).

316 Those taxa might act as degraders of soluble organic matter and provide electroactive microbes

317 simple substrates (Tan et al. 2012, Zeppilli et al. 2020). In addition to fermentative bacteria,

318 methanogens were observed with considerable abundance in S48 (Figure 2) and possibly carried 
319 out syntrophic electron transfer with ethanol-consuming in the presence of conductive granular

320 activated carbon (Yuan et al. 2018).

321

322 Overall, microbial community analysis demonstrated a core BES population composed of primary

323 fermentative bacteria that convert complex organic matter to simple electron donors, and

324 electroactive microbes and their competitors (e.g., methanogens) growing on the fermentative

325 products. The results thus justify the modeling of BES based on those three guilds (Pinto et al.

326 2011). However, such a model structure is incapable of differentiating the contribution of different

327 types of electroactive microbes and their EET mechanisms (i.e., direct contact vs. electron

328 shuttling) due to the experimental challenge to measure the associated biochemical parameters

329 (e.g., the concentration of phenazines and other electron shuttles). The same pitfall is also found

330 in mechanistic modeling of activated sludge and anaerobic digestion, in which the core populations

331 consist of functionally redundant taxa occupying the same ecological niches (Ju and Zhang 2015).

332 This explains the constant parameter calibration required by mechanistic models. To address the

333 issue and improve the prediction robustness, a new modeling approach is imperative.

335 3.3 Reconstruction of microbial community

336 Two types of Bayesian networks were trained with the same dataset containing environmental

337 parameters and relative abundance of the core populations (SI Figure S4-S6): primary networks

338 whose node directions were not restricted by a priori knowledge and directed networks in which

339 the operating parameters were set as the parent nodes. The latter were constructed based on the

340 fact that operating parameters such as external resistance and hydraulic retention time remained 
341 unchanged throughout the operation and hence should be not affected by microbial community

342 dynamics and system performance.

344 To validate the modeling approach and evaluate the prediction of the community structure, Bray-

345 Curtis similarity between the predicted and observed communities was calculated. At the genus

346 level, the directed network achieved the most accurate prediction, followed by the primary network

347 and a null model (Bray-Curtis similarity: $0.72>0.64>0.52, \mathrm{p}<0.05$, SI Figure S7A). Similar

348 trends were observed at the order and phylum levels, but the prediction accuracy did not show

349 consistent improvement as the taxonomic level increased. The Bray-Curtis similarity from the

350 directed networks dropped slightly to 0.61 at the order level and raised back to 0.79 at the phylum

351 level. The results were not in agreement with the previous findings that prediction accuracy could

352 be continuously improved by training data-driven models at higher taxonomic levels (Kuang et al.

353 2016, Yuan et al. 2017). It should be noted that those models were built based on highly specific

354 environments and communities (e.g., acid mine drainage and microbial desalination cells),

355 whereas the models in the present study considered a variety of environmental conditions, which

356 might statistically compromise the model robustness (Walpole et al. 2017).

358 To further validate the modeling approach, relative RMSE was calculated for the 6 environmental

359 parameters and 38 core genera (SI Figure S8-S10). At the genus level, the RMSE values from the

360 directed and primary network were $2 \%-17 \%$ and $3 \%-24 \%$, respectively. The abundances of

361 putative electroactive taxa Desulfovibrio, Pseudomonas, and Acinetobacter were well estimated

362 by both networks with the RMSE ranging from $4 \%$ to $10 \%$. On the other hand, the RMSE for

363 Geobacter was improved from $24 \%$ with the primary network to $16 \%$ with the directed network. 
364 The poor prediction of Geobacter is likely because some members from this genus, despite their

365 dominance in many anaerobic environments (Lee et al. 2016, Lin et al. 2017), are inefficient in or

366 incapable of EET (Lovley et al. 2011, Rotaru et al. 2015). Similar to Bray-Curtis similarity, RMSE

367 was improved at the phylum but not at the order level (SI Figure S9 and S10). The phylum

368 Proteobacteria, which includes the putative electroactive taxa discussed above, is estimated with

369 the highest accuracy (relative RMSE $<1 \%$ ). Overall, the more accurate prediction from the directed

370 networks at all three taxonomic levels suggests that the modeling approach can be enhanced by

371 introducing reasonable structure control.

372

373 After the modeling approach was validated with Bray-Curtis similarity and RMSE, final networks

374 were constructed from the whole dataset to infer microbial interactions (SI Figure S4-S6). In the

375 genus-level networks, putative electroactive taxa Geobacter (G29), Desulfovibrio (G28), and

376 Pseudomonas (G33) did not show any association with CE and current, whilst Acinetobacter (G32)

377 was not correlated with the system output. The networks at higher taxonomic levels yielded even

378 less interpretable inference regarding the potential functions of the core taxa. For example,

379 methanogens were predicted to be more related to current production than Proteobacteria (SI

380 Figure S6A). The results collectively indicate that more a priori knowledge needs to be included

381 in model training to improve the robustness and interpretability of the inference.

\section{$383 \quad 3.4$ Hybrid modeling of BES performance}

384 To build a hybrid model, the rates for substrate utilization and microbial growth and mediator yield

385 were first estimated using the three-population mechanistic component (SI Table S4). Some of the

386 estimates were constant during calibration (e.g., 15 /d for substrate utilization rate) because they 
387 were the boundary values determined based on previous studies (Bruce and Perry 2001, Kato

388 Marcus et al. 2007, Wilson and Kim 2016). The estimated microbial kinetic parameters were

389 subsequently included in the training dataset to construct a hybrid network at the genus level

390 (Figure 3). It should be noted that the scaled kinetic parameters represent the trend of microbial

391 activity and thus do not require accurate estimation or validation. A whitelist function was further

392 applied to force putative electroactive genera Geobacter, Desulfovibrio, Pseudomonas, and

393 Acinetobacterto to directly affect current generation and improve the prediction interpretability.

394 The hybrid network yielded a simulated community that shared a Bray-Curtis similarity of 0.72

395 with the actual genera-level core population, which was comparable to the result from the directed

396 network and significantly better than that of the null model ( $\mathrm{t}$-test, $\mathrm{p}<0.05$ ). The relative RMSE

397 of the hybrid model ranging from $3 \%$ to $18 \%$ was also similar to those from the directed network.

399 The hybrid network generated reasonable inference of the relationships between microbial 400 population and kinetics (Figure 3), as evidenced by the strong positive correlation of the EET401 related substrate utilization rate with Desulfovibrio (coefficient $=0.86$ ), as well as the positive 402 correlation of mediator yield with Pseudomonas. Meanwhile, mediator yield was negatively 403 related to glucose, likely because fermentable substrates could lead to significant electron loss 404 (Parameswaran et al. 2010). The EET-related substrate utilization and growth rates were both 405 associated with the genera (G6 \& G8) from the class Bacteroidia. As discussed above, those taxa 406 can degrade dead cells and soluble microbial products, thereby creating a favorable environment 407 for electroactive microbes ( $\mathrm{Ni}$ et al. 2011, $\mathrm{Ni}$ et al. 2010). Despite those biologically sound 408 inferences, the hybrid model still contained unexplainable interactions such as the negative 
association between the EET-related substrate utilization rate and anode area, underpinning the

410 elimination of data-driven models in prediction interpretability.

412 The developed models were examined with six new samples that were not included in network

413 training, and the hybrid model (hybrid network + mechanistic component) achieved the most

414 accurate prediction of current production compared with the data-driven models. It can be seen

415 from Figure 4 that the predicted results from the hybrid model agree well with the experimental

416 values with slight deviation at the high current range. The low relative error-based RMSE of 0.8

417 further indicates outstanding prediction accuracy throughout the examined current range. The

418 hybrid network alone loses the prediction power at high current, resulting in a higher relative error-

419 based RMSE of 6.7, whereas the directed network is incapable of generating satisfactory prediction

420 and shows the highest relative error-based RMSE of 16.3. The significantly improved prediction

421 performance of the hybrid model likely stems from the close connection between population

422 dynamics and microbial kinetics. Under a given condition, each population (either a single species

423 or a functional guild) has specific maximum substrate utilization and growth rates that are largely

424 determined by its unique lifestyle and ecophysiology (Rittmann and McCarty 2012), which can

425 thus be statistically inferred from the genomic data (Weissman et al. 2021). On the other hand,

426 system performance such as current production is affected by not only microbial population and

427 activity, but also many other operating parameters including electrolyte conductivity and external

428 resistance. Data-driven models that infer system performance directly from the microbial

429 population do not consider the contribution of those operating parameters and hence cannot

430 consistently yield accurate predictions. 
432 Despite the robust performance, the hybrid model is not ready for practical implementation as

433 accurate prediction can only be obtained with microbial community information as the input.

434 When the data-driven component is fed solely with operating parameters, and the simulated

435 microbial community serves as the intermediate to estimate the kinetic parameters, the prediction

436 error quickly builds up along the inference, causing considerable uncertainty to the final prediction.

437 Another challenge is that inadequate biochemical and sequencing data from the selected 438 publications compromise the compatibility of both the data-driven and mechanistic components.

439 These issues will be addressed in future studies with proper experimental design and alternative

440 machine learning algorithms such as neural networks and random forest. Ultimately, the hybrid

441 modeling approach is expected to be broadly applicable to various engineered bioprocesses

442 including anaerobic digesters, activated sludge processes, anaerobic ammonium oxidation, etc.

\section{4. Conclusion}

445 We collected 77 samples from 13 studies in which the BES were operated under diverse conditions.

446 Community analysis revealed a core population composed of primary fermentative bacteria,

447 putative electroactive taxa Geobacter, Desulfovibrio, Pseudomonas, and Acinetobacter, as well as

448 non-electroactive microbes such as methanogens. Bayesian networks were trained with the core

449 populations and validated with Bray-Curtis similarity, relative RMSE, and a null model, all based

450 on a leave-one-out cross-validation strategy. A hybrid model was built by combining mechanistic

451 modeling and network training and achieved more accurate prediction of current production than

452 data-driven models. This study provides insights into incorporating genomic data into hybrid

453 modeling for robust and interpretable prediction. 
bioRxiv preprint doi: https://doi.org/10.1101/2021.04.15.440059; this version posted April 15, 2021. The copyright holder for this preprint (which was not certified by peer review) is the author/funder. All rights reserved. No reuse allowed without permission.

\section{Acknowledgement}

456 This work was supported by the U.S. Department of Agriculture [Award No. 2020-67019-31027]. 


\section{Reference}

Aulenta, F., Catapano, L., Snip, L., Villano, M. and Majone, M. (2012) Linking Bacterial Metabolism to Graphite Cathodes: Electrochemical Insights into the H2-Producing Capability of Desulfovibrio sp. ChemSusChem 5(6), 1080-1085.

Batstone, D.J., Keller, J., Angelidaki, I., Kalyuzhnyi, S., Pavlostathis, S., Rozzi, A., Sanders, W., Siegrist, H. and Vavilin, V. (2002) The IWA anaerobic digestion model no 1 (ADM1). Water Science and Technology 45(10), 65-73.

Bernard, O., Chachuat, B., Hélias, A. and Rodriguez, J. (2006) Can we assess the model complexity for a bioprocess: theory and example of the anaerobic digestion process. Water Science and Technology 53(1), 85-92.

Bhat, N. and McAvoy, T.J. (1990) Use of neural nets for dynamic modeling and control of chemical process systems. Computers \& Chemical Engineering 14(4-5), 573-582.

Bishop, C.M. (2013) Neural networks for pattern recognition, Oxford University Press, Oxford.

Bro, R., Kjeldahl, K., Smilde, A.K. and Kiers, H.A. (2008) Cross-validation of component models: a critical look at current methods. Anal Bioanal Chem 390(5), 1241-1251.

Bruce, E.R. and Perry, L.M. (2001) Environmental Biotechnology: Principles and Applications, McGraw-Hill Education, New York.

Callahan, B.J., McMurdie, P.J., Rosen, M.J., Han, A.W., Johnson, A.J.A. and Holmes, S.P. (2016) DADA2: High-resolution sample inference from Illumina amplicon data. Nature Methods 13(7), 581-583.

Cote, M., Grandjean, B.P., Lessard, P. and Thibault, J. (1995) Dynamic modelling of the activated sludge process: improving prediction using neural networks. Water research 29(4), 995-1004.

De Clercq, D., Jalota, D., Shang, R., Ni, K., Zhang, Z., Khan, A., Wen, Z., Caicedo, L. and Yuan, K. (2019) Machine learning powered software for accurate prediction of biogas production: A case study on industrial-scale Chinese production data. Journal of Cleaner Production 218, 390-399.

DeSantis, T.Z., Hugenholtz, P., Larsen, N., Rojas, M., Brodie, E.L., Keller, K., Huber, T., Dalevi, D., Hu, P. and Andersen, G.L. (2006) Greengenes, a Chimera-Checked 16S rRNA Gene Database and Workbench Compatible with ARB. Applied and Environmental Microbiology 72(7), 5069.

Dongowski, G., Lorenz, A. and Anger, H. (2000) Degradation of Pectins with Different Degrees of Esterification by \&lt;em\&gt;Bacteroides thetaiotaomicron\&lt;/em\&gt; Isolated from Human Gut Flora. Applied and Environmental Microbiology 66(4), 1321. 
Donoso-Bravo, A., Mailier, J., Martin, C., Rodríguez, J., Aceves-Lara, C.A. and Wouwer, A.V. (2011) Model selection, identification and validation in anaerobic digestion: a review. Water research 45(17), 5347-5364.

Gacitúa, M.A., González, B., Majone, M. and Aulenta, F. (2014) Boosting the electrocatalytic activity of Desulfovibrio paquesii biocathodes with magnetite nanoparticles. International Journal of Hydrogen Energy 39(27), 14540-14545.

498 Girvan, M.S., Campbell, C.D., Killham, K., Prosser, J.I. and Glover, L.A. (2005) Bacterial 499 diversity promotes community stability and functional resilience after perturbation.

500 Environmental Microbiology 7(3), 301-313.

501 Gotelli, N.J., \&amp; Graves, G. R. (2002 ) Null models in ecology, Smithsonian Institution 502 Press, Washington.

503 Grenier, D., Mayrand, D. and McBride, B.C. (1989) Further studies on the degradation of 504 immunoglobulins by black-pigmented Bacteroides. Oral Microbiology and Immunology 4(1), $505 \quad 12-18$.

Hensgens, C.M., Vonck, J., Van Beeumen, J., van Bruggen, E.F. and Hansen, T.A. (1993) Purification and characterization of an oxygen-labile, NAD-dependent alcohol dehydrogenase from Desulfovibrio gigas. Journal of Bacteriology 175(10), 2859.

Henze, M., Gujer, W., Mino, T. and van Loosdrecht, M.C. (2000) Activated sludge models ASM1, ASM2, ASM2d and ASM3, IWA publishing.

Jeppsson, U. (1996) Modelling aspects of wastewater treatment processes.

Ju, F. and Zhang, T. (2015) Bacterial assembly and temporal dynamics in activated sludge of a full-scale municipal wastewater treatment plant. The ISME Journal 9(3), 683-695.

Kahn, S.D. (2011) On the future of genomic data. Science 331(6018), 728-729.

Karama, A., Bernard, O., Gouzé, J., Benhammou, A. and Dochain, D. (2001) Hybrid neural

517 Technology 43(7), 1-8.

518 Kato Marcus, A., Torres, C.I. and Rittmann, B.E. (2007) Conduction-based modeling of the 519 biofilm anode of a microbial fuel cell. Biotechnology and Bioengineering 98(6), 1171-1182.

520 Kremer, D.R., Nienhuis-Kuiper, H.E. and Hansen, T.A. (1988) Ethanol dissimilation in 521 Desulfovibrio. Archives of Microbiology 150(6), 552-557.

522 Kuang, J., Huang, L., He, Z., Chen, L., Hua, Z., Jia, P., Li, S., Liu, J., Li, J., Zhou, J. and Shu, W. 523 (2016) Predicting taxonomic and functional structure of microbial communities in acid mine 524 drainage. The ISME Journal 10(6), 1527-1539. 
Larsen, P.E., Field, D. and Gilbert, J.A. (2012) Predicting bacterial community assemblages using an artificial neural network approach. Nature Methods 9(6), 621-625.

527 Lee, D.S., Jeon, C.O., Park, J.M. and Chang, K.S. (2002) Hybrid neural network modeling of a full-scale industrial wastewater treatment process. Biotechnology and Bioengineering 78(6), 670-682.

530 Lee, H.-S., Torres, C.I. and Rittmann, B.E. (2009) Effects of Substrate Diffusion and Anode 531 Potential on Kinetic Parameters for Anode-Respiring Bacteria. Environmental Science \& 532 Technology 43(19), 7571-7577.

Lee, J.-Y., Lee, S.-H. and Park, H.-D. (2016) Enrichment of specific electro-active microorganisms and enhancement of methane production by adding granular activated carbon in anaerobic reactors. Bioresource Technology 205, 205-212.

536 Lesnik, K.L., Cai, W. and Liu, H. (2020) Microbial Community Predicts Functional Stability of 537 Microbial Fuel Cells. Environmental Science \& Technology 54(1), 427-436.

538 Lesnik, K.L. and Liu, H. (2017) Predicting Microbial Fuel Cell Biofilm Communities and Bioreactor Performance using Artificial Neural Networks. Environmental Science \& Technology 51(18), 10881-10892.

Lin, R., Cheng, J., Zhang, J., Zhou, J., Cen, K. and Murphy, J.D. (2017) Boosting biomethane yield and production rate with graphene: The potential of direct interspecies electron transfer in anaerobic digestion. Bioresource Technology 239, 345-352. populations and seasonality of water meter biofilms in a metropolitan drinking water distribution system. The ISME Journal 10(3), 582-595. (2013) Extracellular Electron Transfer of a Highly Adhesive and Metabolically Versatile Bacterium. ChemPhysChem 14(11), 2407-2412. modeling approach to direct interspecies electron transfer process in anaerobic transformation of ethanol to methane. Environmental Science and Pollution Research 24(1), 855-863. Technology 41(9), 3341-3346. 
Lovley, D.R., Ueki, T., Zhang, T., Malvankar, N.S., Shrestha, P.M., Flanagan, K.A., Aklujkar, M., Butler, J.E., Giloteaux, L., Rotaru, A.-E., Holmes, D.E., Franks, A.E., Orellana, R., Risso, C. and Nevin, K.P. (2011) Advances in Microbial Physiology. Poole, R.K. (ed), pp. 1-100, Academic Press.

Ludwig, W., Strunk, O., Westram, R., Richter, L., Meier, H., Yadhukumar, Buchner, A., Lai, T., Steppi, S., Jobb, G., Förster, W., Brettske, I., Gerber, S., Ginhart, A.W., Gross, O., Grumann, S., Hermann, S., Jost, R., König, A., Liss, T., Lüßmann, R., May, M., Nonhoff, B., Reichel, B., Strehlow, R., Stamatakis, A., Stuckmann, N., Vilbig, A., Lenke, M., Ludwig, T., Bode, A. and Schleifer, K.H. (2004) ARB: a software environment for sequence data. Nucleic Acids Research 32(4), 1363-1371.

Madigan, M., Bender, K., Buckley, D., Sattley, W. and Stahl, D. (2014) Brock biology of microorganisms Pearson, Boston.

Mendes, C., da Silva Magalhes, R., Esquerre, K. and Queiroz, L.M. (2015) Artificial Neural Network Modeling for Predicting Organic Matter in a Full-Scale Up-Flow Anaerobic Sludge Blanket (UASB) Reactor. Environmental Modeling \& Assessment 20(6), 625-635.

Miyahara, M., Kouzuma, A. and Watanabe, K. (2015) Effects of NaCl concentration on anode microbes in microbial fuel cells. AMB Express 5(1), 34.

Moral, H., Aksoy, A. and Gokcay, C.F. (2008) Modeling of the activated sludge process by using artificial neural networks with automated architecture screening. Computers \& Chemical Engineering 32(10), 2471-2478.

Ng, A.N.L. and Kim, A.S. (2007) A mini-review of modeling studies on membrane bioreactor (MBR) treatment for municipal wastewaters. Desalination 212(1), 261-281.

Ni, B.-J., Rittmann, B.E. and Yu, H.-Q. (2011) Soluble microbial products and their implications in mixed culture biotechnology. TRENDS in Biotechnology 29(9), 454-463.

Ni, B.-J., Zeng, R.J., Fang, F., Xie, W.-M., Sheng, G.-P. and Yu, H.-Q. (2010) Fractionating soluble microbial products in the activated sludge process. Water research 44(7), 2292-2302. significance of hydrogen scavengers. Biotechnology and Bioengineering 105(1), 69-78.

590 Ping, Q., Zhang, C., Chen, X., Zhang, B., Huang, Z. and He, Z. (2014) Mathematical Model of 591 Dynamic Behavior of Microbial Desalination Cells for Simultaneous Wastewater Treatment and 592 Water Desalination. Environmental Science \& Technology 48(21), 13010-13019.

593 Pinto, R.P., Srinivasan, B., Escapa, A. and Tartakovsky, B. (2011) Multi-Population Model of a 594 Microbial Electrolysis Cell. Environmental Science \& Technology 45(11), 5039-5046.

595 Pinto, R.P., Srinivasan, B., Manuel, M.F. and Tartakovsky, B. (2010) A two-population bio596 electrochemical model of a microbial fuel cell. Bioresource Technology 101(14), 5256-5265. 
Quast, C., Pruesse, E., Yilmaz, P., Gerken, J., Schweer, T., Yarza, P., Peplies, J. and Glöckner, F.O. (2013) The SILVA ribosomal RNA gene database project: improved data processing and web-based tools. Nucleic Acids Research 41(D1), D590-D596. Select for Microbial Consortia That Self-Mediate Electron Transfer. Applied and Environmental Microbiology 70(9), 5373.

603 Reichenbach, H. (1992) The Prokaryotes: A Handbook on the Biology of Bacteria:

604 Ecophysiology, Isolation, Identification, Applications. Balows, A., Trüper, H.G., Dworkin, M.,

605 Harder, W. and Schleifer, K.-H. (eds), pp. 3631-3675, Springer New York, New York, NY.

Rittmann, B.E. and McCarty, P.L. (2012) Environmental biotechnology: principles and

608 Rodríguez, J., Roca, E., Lema, J.M. and Bernard, O. (2008) Determination of the adequate minimum model complexity required in anaerobic bioprocesses using experimental data. Journal of Chemical Technology \& Biotechnology: International Research in Process, Environmental \& 611 Clean Technology 83(12), 1694-1702.

612 Rognes, T., Flouri, T., Nichols, B., Quince, C. and Mahé, F. (2016) VSEARCH: a versatile open source tool for metagenomics. PeerJ 4, e2584.

614 Rotaru, A.-E., Woodard, T.L., Nevin, K.P. and Lovley, D.R. (2015) Link between capacity for 615 current production and syntrophic growth in Geobacter species. Frontiers in Microbiology 616 6(744).

617 Rudin, C. (2019) Stop explaining black box machine learning models for high stakes decisions 618 and use interpretable models instead. Nature Machine Intelligence 1(5), 206-215.

619 Saunders, A.M., Albertsen, M., Vollertsen, J. and Nielsen, P.H. (2016) The activated sludge 620 ecosystem contains a core community of abundant organisms. The ISME Journal 10(1), 11-20.

621 Scutari, M. (2010) Learning Bayesian Networks with the bnlearn R Package. Journal of 622 Statistical Software. Journal of Statistical Software 35(3), 1-22.

623 Tan, H.-Q., Li, T.-T., Zhu, C., Zhang, X.-Q., Wu, M. and Zhu, X.-F. (2012) Parabacteroides 624 chartae sp. nov., an obligately anaerobic species from wastewater of a paper mill. International 625 Journal of Systematic and Evolutionary Microbiology 62(Pt_11), 2613-2617.

626 Torres, C.I., Marcus, A.K., Lee, H.-S., Parameswaran, P., Krajmalnik-Brown, R. and Rittmann, 627 B.E. (2010) A kinetic perspective on extracellular electron transfer by anode-respiring bacteria. 628 FEMS Microbiology Reviews 34(1), 3-17.

629 Torres, C.I., Marcus, A.K., Parameswaran, P. and Rittmann, B.E. (2008) Kinetic Experiments for 630 Evaluating the Nernst-Monod Model for Anode-Respiring Bacteria (ARB) in a Biofilm Anode. 631 Environmental Science \& Technology 42(17), 6593-6597. 
632 Uusitalo, L. (2007) Advantages and challenges of Bayesian networks in environmental

633 modelling. Ecological Modelling 203(3), 312-318.

634 Walpole, R.E., Myers, R.H., Myers, S.L. and Ye, K. (2017) Probability \& statistics for engineers

$635 \&$ scientists, Pearson Education South Asia Pte, Singapore.

636 Wang, B., Liu, W., Zhang, Y. and Wang, A. (2020a) Bioenergy recovery from wastewater 637 accelerated by solar power: Intermittent electro-driving regulation and capacitive storage in 638 biomass. Water Research 175, 115696.

639 Wang, H. and Ren, Z.J. (2013) A comprehensive review of microbial electrochemical systems as 640 a platform technology. Biotechnology Advances 31(8), 1796-1807.

641 Wang, L., Long, F., Liao, W. and Liu, H. (2020b) Prediction of anaerobic digestion performance 642 and identification of critical operational parameters using machine learning algorithms.

643 Bioresource Technology 298, 122495.

644 Weissman, J.L., Hou, S. and Fuhrman, J.A. (2021) Estimating maximal microbial growth rates 645 from cultures, metagenomes, and single cells via codon usage patterns. Proceedings of the 646 National Academy of Sciences 118(12), e2016810118.

647 Wilson, E.L. and Kim, Y. (2016) The yield and decay coefficients of exoelectrogenic bacteria in 648 bioelectrochemical systems. Water research 94, 233-239.

649 Yates, M.D., Kiely, P.D., Call, D.F., Rismani-Yazdi, H., Bibby, K., Peccia, J., Regan, J.M. and 650 Logan, B.E. (2012) Convergent development of anodic bacterial communities in microbial fuel 651 cells. The ISME Journal 6(11), 2002-2013.

652 Yu, L., Duan, J., Zhao, W., Huang, Y. and Hou, B. (2011) Characteristics of hydrogen evolution 653 and oxidation catalyzed by Desulfovibrio caledoniensis biofilm on pyrolytic graphite electrode. 654 Electrochimica Acta 56(25), 9041-9047.

655 Yuan, H.-Y., Ding, L.-J., Zama, E.F., Liu, P.-P., Hozzein, W.N. and Zhu, Y.-G. (2018) Biochar 656 Modulates Methanogenesis through Electron Syntrophy of Microorganisms with Ethanol as a 657 Substrate. Environmental Science \& Technology 52(21), 12198-12207.

658 Yuan, H., Abu-Reesh, I.M. and He, Z. (2016) Mathematical Modeling Assisted Investigation of 659 Forward Osmosis as Pretreatment for Microbial Desalination Cells to Achieve Continuous Water 660 Desalination and Wastewater Treatment. Journal of Membrane Science 502, 116-123.

661 Yuan, H., Lu, Y., Abu-Reesh, I. and He, Z. (2015) Bioelectrochemical production of hydrogen 662 in an innovative pressure-retarded osmosis/microbial electrolysis cell system: experiments 663 and modeling. Biotechnology for Biofuels 8(116), 1-12.

664 Yuan, H., Mei, R., Liao, J. and Liu, W.-T. (2019) Nexus of Stochastic and Deterministic 665 Processes on Microbial Community Assembly in Biological Systems. Frontiers in Microbiology 666 10(1536). 
667 Yuan, H., Sun, S., Abu-Reesh, I.M., Badgley, B.D. and He, Z. (2017) Unravelling and

668 Reconstructing the Nexus of Salinity, Electricity, and Microbial Ecology for Bioelectrochemical

669 Desalination. Environmental Science \& Technology 51(21), 12672-12682.

670 Zendehboudi, S., Rezaei, N. and Lohi, A. (2018) Applications of hybrid models in chemical, 671 petroleum, and energy systems: A systematic review. Applied Energy 228, 2539-2566.

672 Zeppilli, M., Chouchane, H., Scardigno, L., Mahjoubi, M., Gacitua, M., Askri, R., Cherif, A. and 673 Majone, M. (2020) Bioelectrochemical vs hydrogenophilic approach for CO2 reduction into 674 methane and acetate. Chemical Engineering Journal 396, 125243.

675 Zhao, H., Hao, O.J., McAvoy, T.J. and Chang, C.-H. (1997) Modeling nutrient dynamics in 676 sequencing batch reactor. Journal of Environmental Engineering 123(4), 311-319.

677 Zhao, X., Li, L., Wu, D., Xiao, T., Ma, Y. and Peng, X. (2019) Modified Anaerobic Digestion 678 Model No. 1 for modeling methane production from food waste in batch and semi-continuous 679 anaerobic digestions. Bioresource Technology 271, 109-117.

680 Zhu, X., Yates, M.D., Hatzell, M.C., Ananda Rao, H., Saikaly, P.E. and Logan, B.E. (2014)

681 Microbial Community Composition Is Unaffected by Anode Potential. Environmental Science \& 682 Technology 48(2), 1352-1358. 


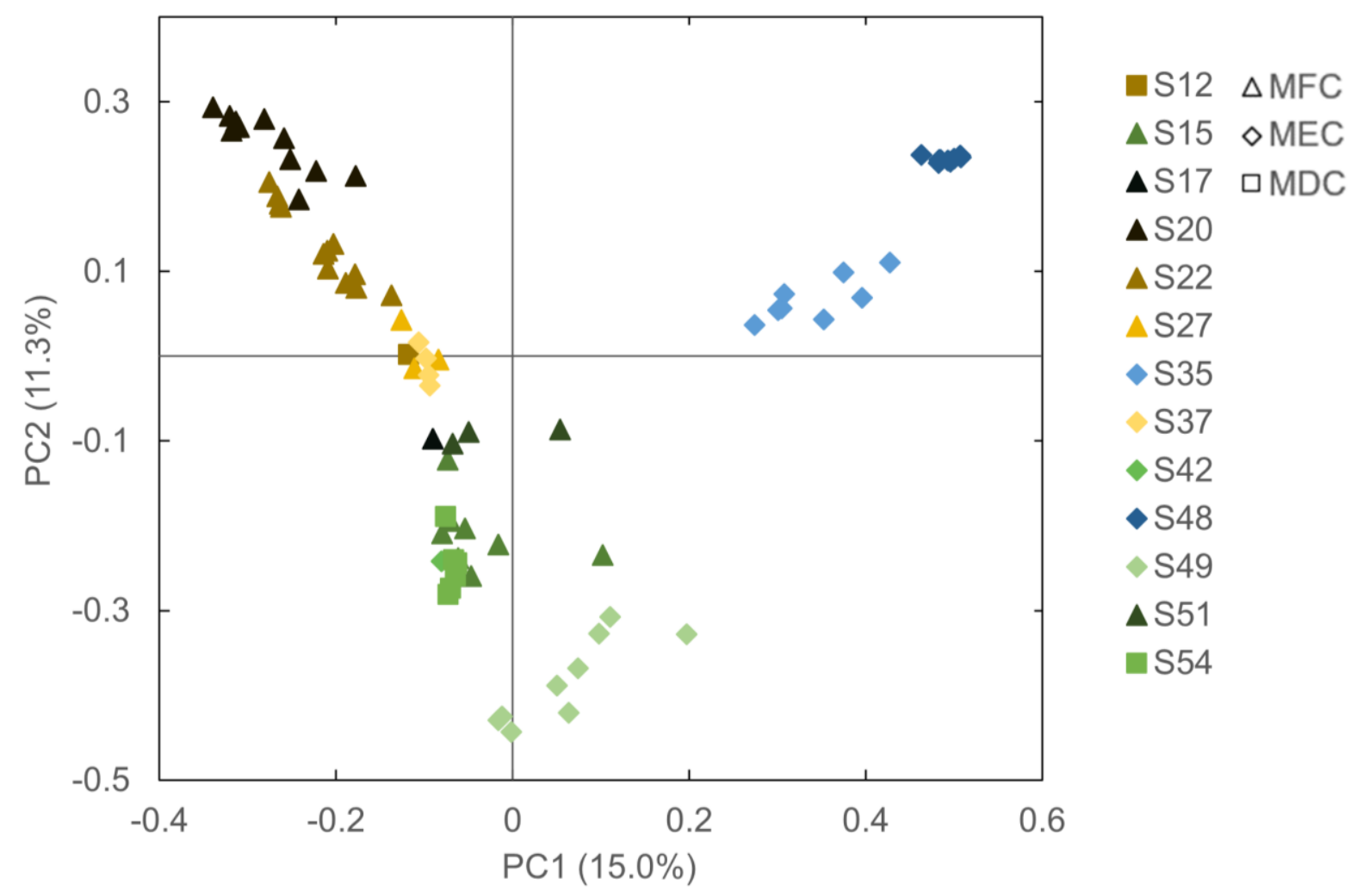

Figure 1. Bray-Curtis distance-based PCoA of the 13 selected studies. MFC: microbial fuel cells, MEC: microbial electrolysis cells, MDC: microbial desalination cells. 


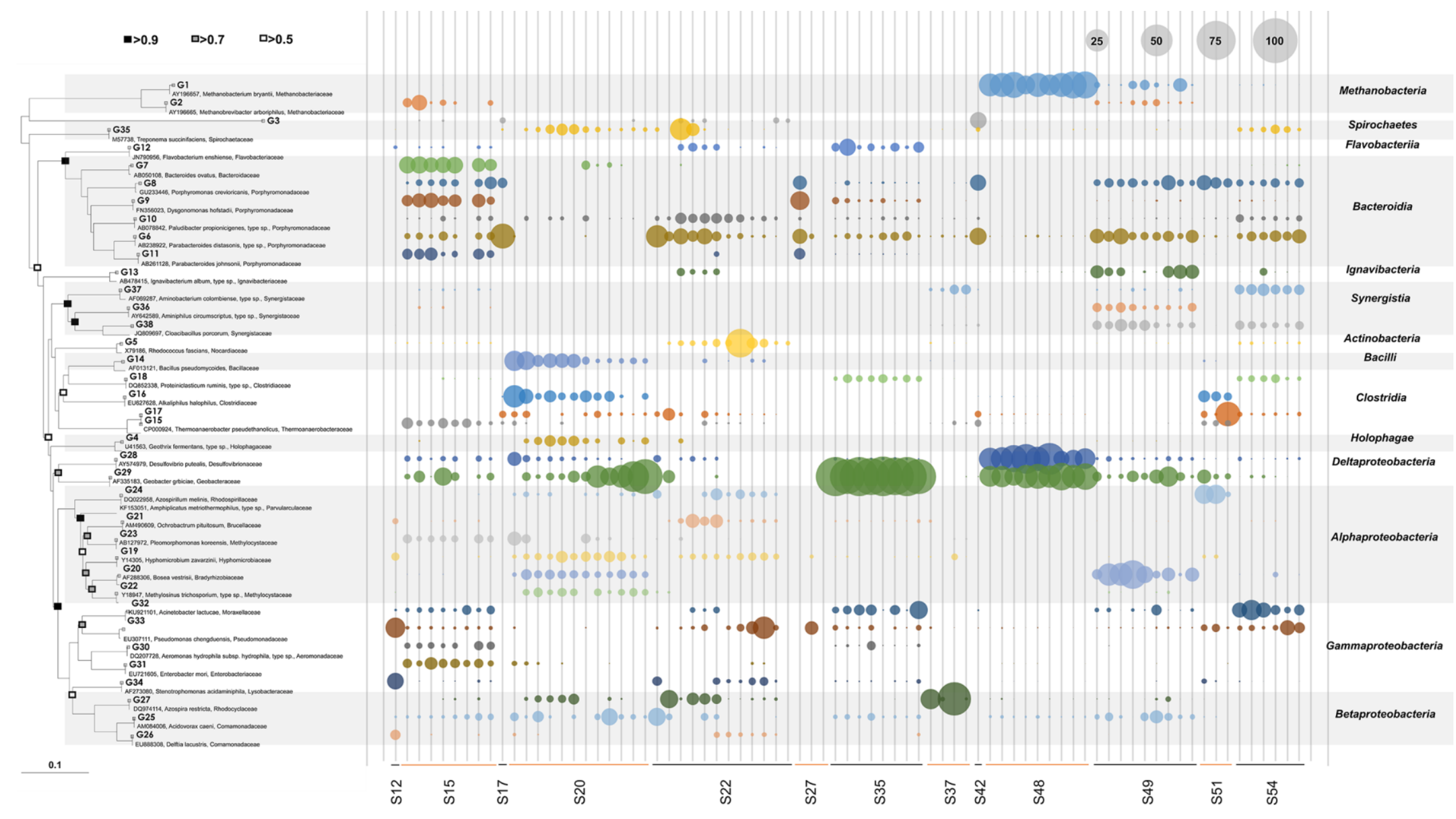

Figure 2. Phylogenetic tree and relative abundance of 38 core genera selected from the 77 samples in 13 publications. 


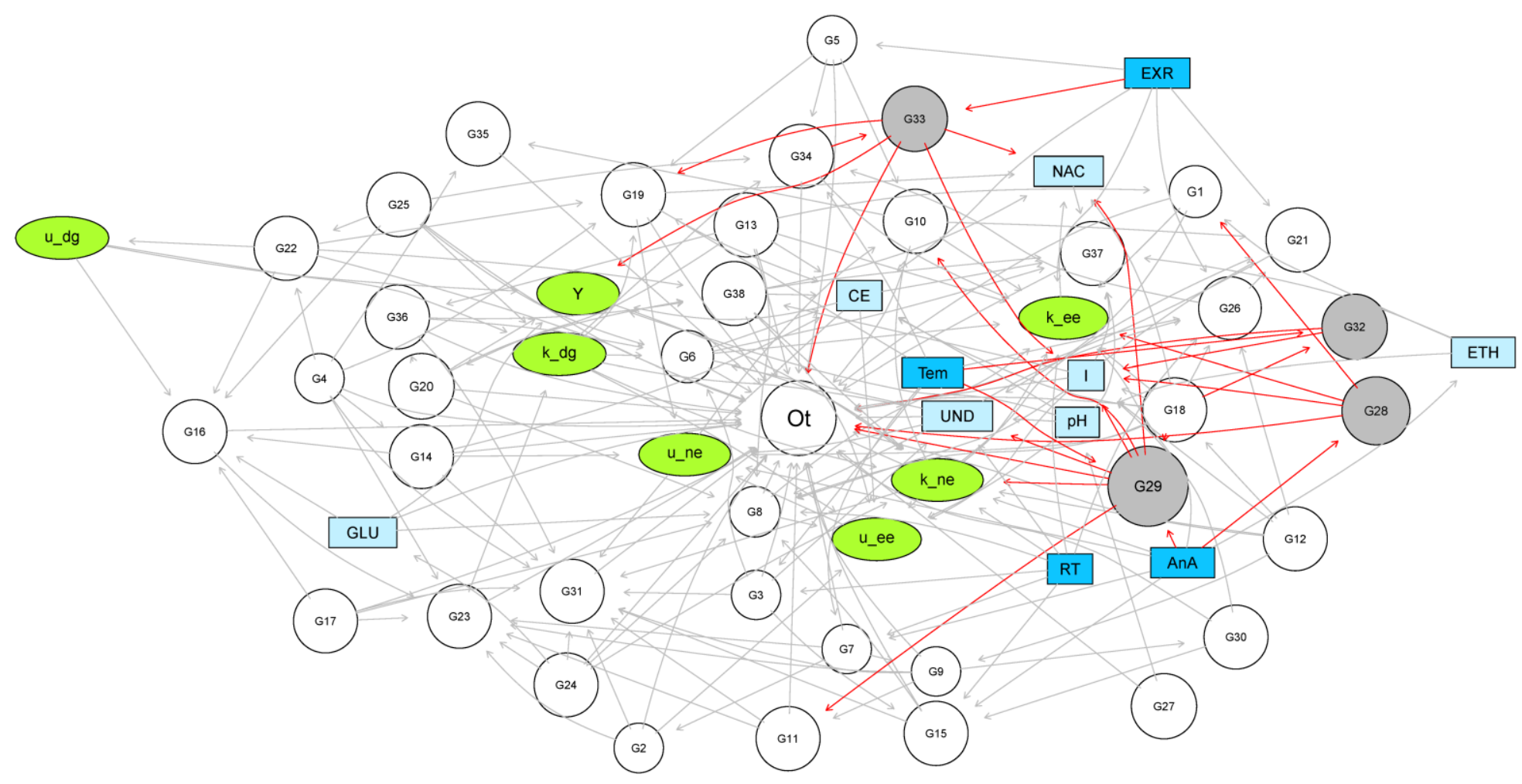

Figure 3. The hybrid Bayesian network at the genus level. Geobacter (G29), Desulfovibrio (G28), Pseudomonas (G33), and Acinetobacter (G32) are highlighted in grey and forced to directly affect current. The symbols for the core genera can be found in SI Table S6. Light blue nodes are biochemical parameters. Dark blue nodes are operating parameters unaffected by other nodes and serve only as the parent nodes. Green nodes are kinetic parameters estimated using the mechanistic component, in which u_dg, $u \_e e$, and $u \_n e$ are the maximum growth rates for primary degraders, electroactive microbes, and non-electroactive microbes, respectively. $\mathrm{k}$ is the maximum substrate utilization rate and $\mathrm{Y}$ is the mediator yield. EXR: external resistance, NAC: acetate, CE: coulombic efficiency, I: current, Tem: temperature, ETH: ethanol, GLU: glucose, RT: hydraulic retention time, AnA: anode area, UND: undefined substrate. 


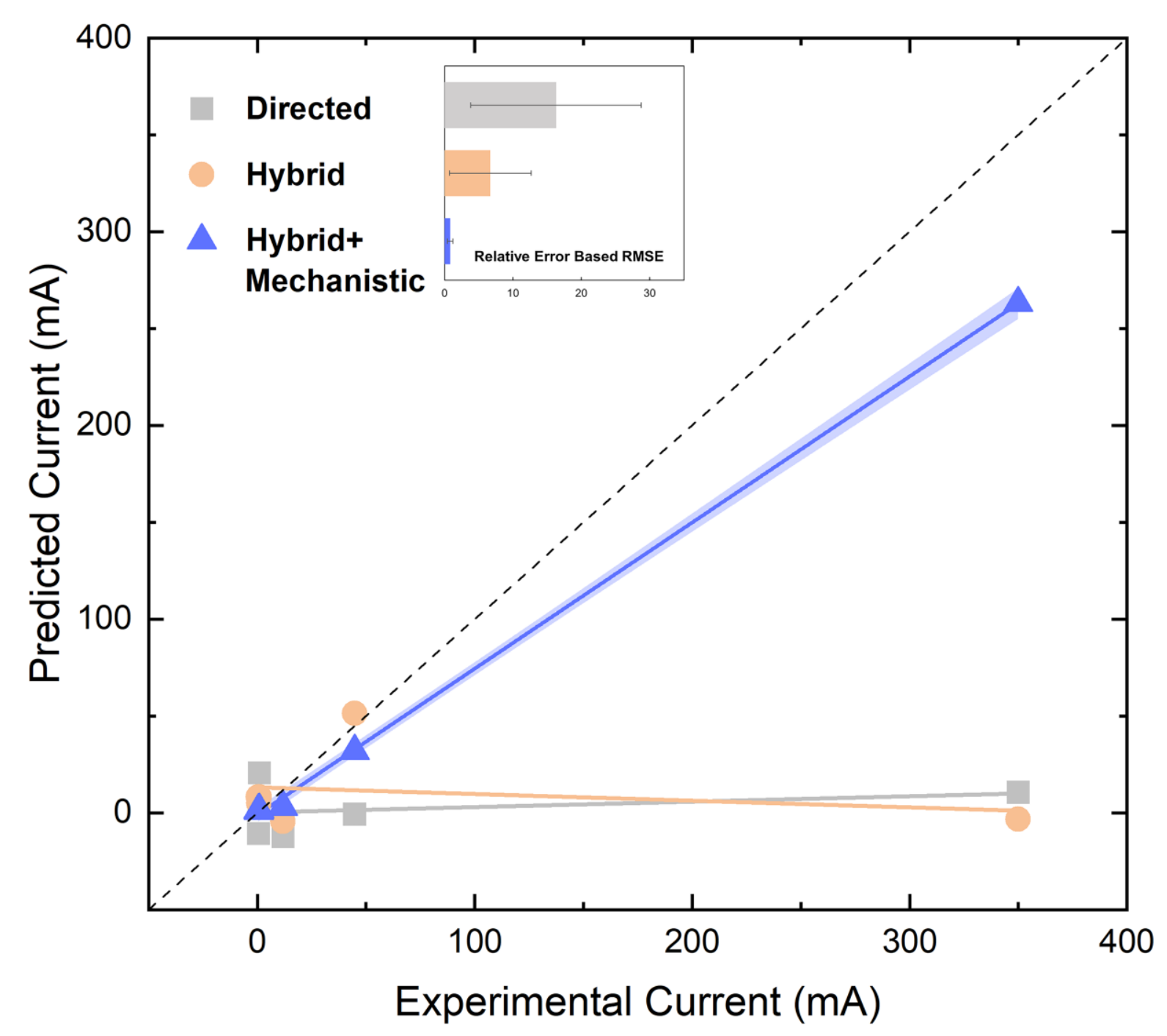

683

Figure 4. Comparison of experimental and predicted current from the directed Bayesian network, hybrid Bayesian network, and hybrid model (hybrid network + mechanistic component). Inset in the relative error-based RMSE. 